\title{
Erratum to: Flow Around a Crack in a Porous Matrix and Related Problems
}

\author{
Ahmad Pouya - Siavash Ghabezloo
}

Published online: 2 March 2010

(C) Springer Science+Business Media B.V. 2010

\section{Erratum to: Transp Porous Med DOI 10.1007/s11242-009-9517-8}

There are three corrections to be made to the text in the original paper:

(I) Equation (34): $Y 2$ must be replaced by $Y^{2}$, so the correct expression is:

$$
p(x, y)=a L X \Phi\left(X^{2}, Y^{2}\right)
$$

(II) Equation (35): There is an $\alpha_{1}$ missing in the denominator, so the correct expression is:

$$
\Phi\left(\alpha_{1}, \alpha_{2}\right)=\sqrt{\frac{\sqrt{\left(1-\alpha_{1}+\alpha_{2}\right)^{2}+4 \alpha_{1} \alpha_{2}}-\left(1-\alpha_{1}+\alpha_{2}\right)}{2 \alpha_{1}}}
$$

(III) Equation (51): the second integral $\int_{\partial \Omega} \mathbf{k} \cdot \underline{\nabla} p \mathrm{~d} s$ must be replaced by $\mathbf{k} \cdot \int_{\partial \Omega} p \underline{n} \mathrm{~d} s$, so the correct expression is:

$$
\frac{1}{\Omega} \int_{\Omega} \underline{v} \mathrm{~d} \omega=-\frac{1}{\Omega} \mathbf{k} \cdot \int_{\partial \Omega} p \underline{n} \mathrm{~d} s=-\mathbf{k} \cdot \underline{A}
$$

The online version of the original article can be found under doi:10.1007/s11242-009-9517-8.

A. Pouya $(\varangle)$

Université Paris-Est, LCPC, MSRGI, 58, Boulevard Lefebvre, Paris 75015, France

e-mail: pouya@lcpc.fr

S. Ghabezloo

Université Paris-Est, UR Navier, CERMES, Ecole des Ponts ParisTech, 6-8 Avenue Blaise Pascal,

Cité Descartes, Champs-sur-Marne, Marne la Vallée Cedex 2, 77455, France 